\title{
Surface morphology effects on clathrate hydrate wettability
}

\author{
Anh Phan ${ }^{\mathrm{a}}$, Hannah M. Stoner ${ }^{\mathrm{b}}$, Michail Stamatakis ${ }^{\mathrm{a}}$, Carolyn A. Koh ${ }^{\mathrm{b}}$, Alberto Striolo ${ }^{\mathrm{a}, \mathrm{c}, *}$ \\ a Department of Chemical Engineering, University College London, London WC1E 7JE, UK \\ ${ }^{\mathrm{b}}$ Center for Hydrate Research, Chemical \& Biological Engineering Department, Colorado School of Mines, Golden, CO 80401, United States \\ ${ }^{\mathrm{c}}$ School of Chemical, Biological and Materials Engineering, University of Oklahoma, Norman, OK 73019, United States
}

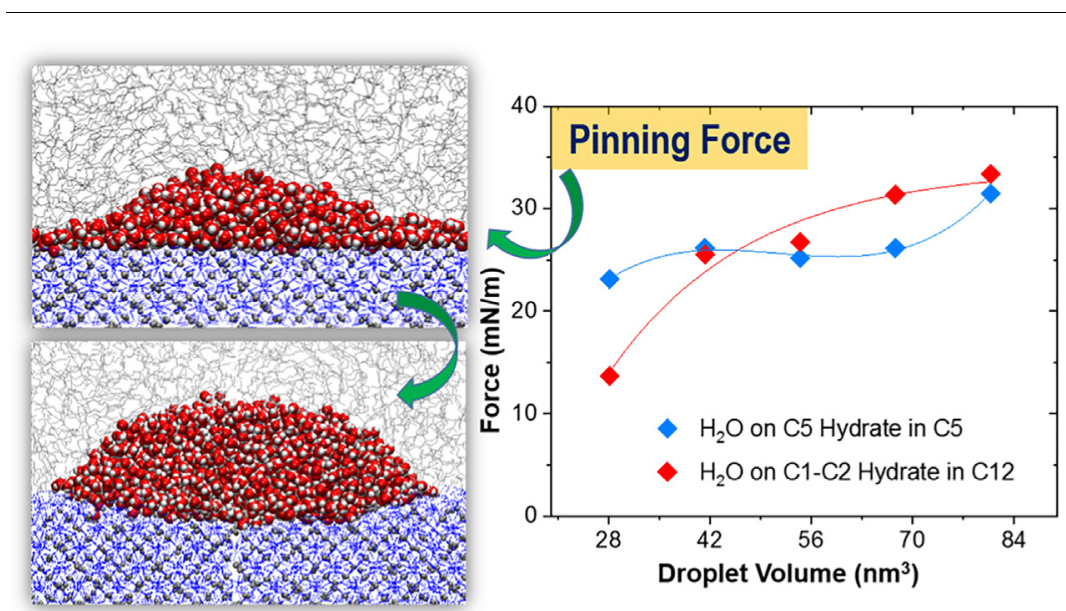

\section{A R T I C L E I N F O}

\section{Article history:}

Received 8 October 2021

Revised 8 December 2021

Accepted 13 December 2021

Available online 16 December 2021

\section{Keywords:}

Contact angle

Pinning force

Work of adhesion

Interfacial tension

Surface heterogeneity

\begin{abstract}
A B S T R A C T
Hypothesis: Clathrate hydrates preferentially form at interfaces; hence, wetting properties play an important role in their formation, growth, and agglomeration. Experimental evidence suggests that the hydrate preparation process can strongly affect contact angle measurements, leading to the different results reported in the literature. These differences hamper technological progress. We hypothesize that changes in hydrate surface morphologies are responsible for the wide variation of contact angles reported in the literature.

Experiments: Experimental testing of our hypothesis is problematic due to the preparation history of hydrates on their surface properties, and the difficulties in advanced surface characterization. Thus, we employ molecular dynamics simulations, which allow us to systematically change the interfacial features and the system composition. Implementing advanced algorithms, we quantify fundamental thermodynamic properties to validate our observations.

Findings: We achieve excellent agreement with experimental observations for both atomically smooth and rough hydrate surfaces. Our results suggest that contact line pinning forces, enhanced by surface heterogeneity, are accountable for altering water contact angles, thus explaining the differences among reported experimental data. Our analysis and molecular level insights help interpret adhesion force measurements and yield a better understanding of the agglomeration between hydrate particles, providing a microscopic tool for advancing flow assurance applications.
\end{abstract}

๑ 2021 The Authors. Published by Elsevier Inc. This is an open access article under the CC BY license (http:// creativecommons.org/licenses/by/4.0/).

\footnotetext{
* Corresponding author at: School of Chemical, Biological and Materials Engineering, University of Oklahoma, Norman, OK 73019.

E-mail addresses: a.striolo@ucl.ac.uk, astriolo@ou.edu (A. Striolo).
} 


\section{Introduction}

Gas hydrates naturally form under certain conditions of pressure and temperature because of a balance between water-water hydrogen bonds and host-guest (i.e., water-methane) dispersive interactions [1]. Over the last decade, gas hydrates have attracted significant focus because of their possible applications in various sustainable and environmental technologies [2-9]. Natural gas hydrates could also provide an alternative energy source; notwithstanding their environmental effects should be understood, prevented and mitigated when necessary $[8,10]$. The uncontrolled formation of hydrate plugs in oil/gas pipelines [1] and offshore energy operations [11] also causes disruptions in oil and gas production, leading to accidents, as well as large negative environmental consequences [12-14].

As the formation of hydrates occurs at interfaces, it has been proposed that hydrate formation and agglomeration could be inhibited by altering the water wettability of hydrates $[15,16]$ using low dosage hydrate inhibitors, thereby preventing the interaction/coalescence among hydrate particles and water droplets $[12,17-20]$. Understanding hydrate wettability is therefore crucial in optimizing flow assurance activities, where a remarkable change is taking place from "hydrate avoidance" to "hydrate management" $[21,22]$. At the macroscopic scale, wetting properties can be assessed by measuring the Young contact angle of a liquid droplet on a rigid and smooth surface [23]. However, this is challenging for hydrates, which form at high-pressure \& low-temperature conditions following processes that are stochastic in nature [24]. Notwithstanding many practical obstacles, recent research advances have been achieved in the direct measurement of contact angles for hydrate systems [16,25]. For example, Brown et al. [16] reported an average water contact angle on a cyclopentane (C5) hydrate in liquid $\mathrm{C} 5$ of $94.2^{\circ} \pm 8.5^{\circ}$ at atmospheric pressure and temperatures of $273-280 \mathrm{~K}$. However, the reported contact angles vary significantly in the literature, perhaps because the preparation of the hydrate surfaces impacts the measured contact angles. For example, in contrast to Brown et al. [16], who used an annealing time of $30 \mathrm{~min}$, Thomas et al. [25] implemented temperature cycling protocols to create C5 hydrates over a period of $\sim 24 \mathrm{~h}$, inspired by the experimental procedures employed by Zylyftari et al. [26] Using the latter substrates, Thomas et al. [25] studied the spreading dynamics of a water droplet and showed a fully wetted C5 hydrate $7 \mathrm{~s}$ after droplet deposition. Supporting the possible effect of preparation on wetting properties, Li et al. [27] showed significant differences in the hydrate surface morphologies when changing gas compositions and degree of subcooling in the process of creating ethane and methane-ethane (C1-C2) hydrates. It is possible that both hydrate porosity and surface roughness affect the spreading dynamics of a water droplet on a hydrate surface, explaining the different results reported in the literature $[16,25,28]$. Reconciling the differences in wetting properties is essential, as these fundamental interfacial properties are related to the cohesive/adhesive forces responsible for hydrate agglomeration and pipelines plugging. It is expected that hydrates formed in practical field applications would differ in morphology and surface features from hydrates formed in the laboratory under controlled physical conditions.

Because of the practical hurdles faced by experimental approaches, we implement here atomistic molecular dynamics (MD) simulations to probe the wetting properties of atomically smooth and rough C5 at atmospheric pressure. As realistic flow assurance activities occur at higher pressure and lower temperature, here we also examine the wettability of $\mathrm{C} 1-\mathrm{C} 2$ hydrates in liquid hydrocarbons under high-pressure \& low-temperature conditions (3.45 MPa and $274 \mathrm{~K}$ ). We quantify contact angles of a water droplet on hydrate surfaces with different morphologies, surface tensions of hydrate-liquid and liquid-liquid interfaces, contact line pinning forces as induced by surface heterogeneity, and work of adhesion (these quantities are defined later) between water droplets and hydrate particles. The simulation results, in general agreement with several experimental observations, help us reconcile at the molecular-level the differences among contact angles reported in the literature, and further highlight the applicability of simulated interfacial properties to examine adhesion force measurements and predict properties of experimental relevance.

In what follows, we first describe the simulation methodologies and force fields implemented, then discuss our simulated results, compare them against a variety of experimental datasets, and summarize our fundamental observations with main practical implications.

\section{Methods}

Model Setup. Our simulation setup (see Fig. 1A) mimics the sessile contact angle measurement for a water droplet on a hydrate surface in the presence of a liquid hydrocarbon. In this study, two systems are considered: (1) a water droplet on a C5 hydrate immersed in bulk liquid C5 at atmospheric pressure and $275 \mathrm{~K}$, and (2) a water droplet on a C1-C2 hydrate surface immersed in bulk liquid n-dodecane (C12) at $274 \mathrm{~K}$ and $3.45 \mathrm{MPa}$. The sII hydrate structure was employed as the solid substrate for both $\mathrm{C} 5$ and $\mathrm{C} 1-\mathrm{C} 2$ hydrates. To construct the hydrate configurations, we adopted the sII unit cell from Takeuchi et al. [29]. Tiling the unit cell in $X$ and $Y$ directions, the $X, Y$ and $Z$ dimensions of the hydrate substrate were $10.386,5.193$, and $1.731 \mathrm{~nm}$, respectively. We carried out simulations with a droplet of 940 water molecules starting from various initial configurations (such as rectangular or cylindrical) placed on the hydrate substrates and surrounded by hydrocarbon ( $\mathrm{C} 5 / \mathrm{C} 12)$ molecules. The resultant simulation box length was $\sim 10.8 \mathrm{~nm}$ in the $Z$ direction, with the hydrate substrate aligned parallel to the $X-Y$ plane. The hydrate substrate model becomes infinite along the $X$ and $Y$ directions when applying periodic boundary conditions in all directions.

Upon equilibration, we used a cylindrical water droplet periodic along the $Y$ direction - the cylinder axis, as this configuration guarantees that the line tension of the three-phase boundary does not influence contact angle estimates from simulations [30,31].

To quantify the hydrate-liquid interfacial free energy, we simulated systems in which a thin film of liquid molecules, i.e., C5, C12, or water, is placed on the hydrate substrate (see Fig. 2A). The number of water, C5, and C12 molecules on the hydrates were 7000 , 2600 , and 1200 , respectively, yielding a water film of $\sim 4 \mathrm{~nm}$ and a liquid hydrocarbon film of $\sim 9 \mathrm{~nm}$ in thickness. In these simulations, we used the same sIl hydrate substrates employed in the contact angle measurements, with their surface parallel to the $X-Y$ plane. Because the length of the simulation box in the $Z$ direction was $17.731 \mathrm{~nm}$, and the thickness of solid substrate was $1.731 \mathrm{~nm}$, there is an empty gap between the periodic image of the hydrate substrate and the thin film [32,33]. To evaluate liquid-liquid interfacial tensions, we conducted simulations for systems composed of the water-hydrocarbon ( $\mathrm{C} 5, \mathrm{C} 12$ or C5toluene) interface at selected temperature $(T)$ and pressure $(P)$ conditions. The water-hydrocarbon interface model was generated by combining aqueous and hydrocarbon phases into $4.0 \times 4.0 \times \sim 13.0$ $\left(\mathrm{nm}^{3}\right)$ simulation boxes, as shown in Figure $\mathbf{S 1}$ in Supporting Information (SI). Additional simulations for systems with waterhydrocarbon interfacial areas of $5.0 \times 5.0 \mathrm{~nm}^{2}$ were conducted to verify that using smaller surface areas $\left(4.0 \times 4.0 \mathrm{~nm}^{2}\right)$ was sufficient to extract reliable liquid-liquid surface tension results. The results were comparable. We have employed similar approaches to quantify the surface tensions of liquid-liquid interfaces for a 
A)

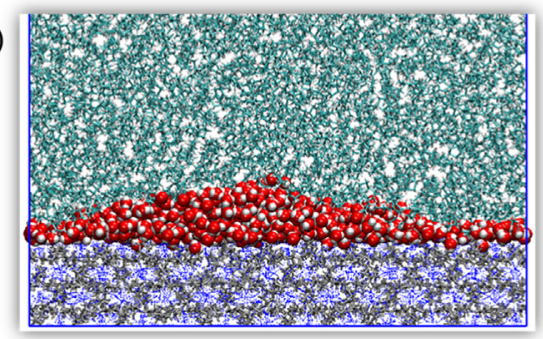

C)

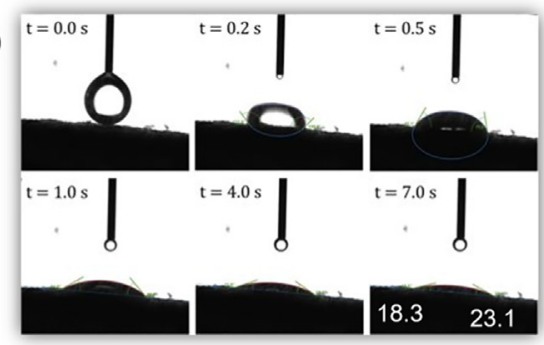

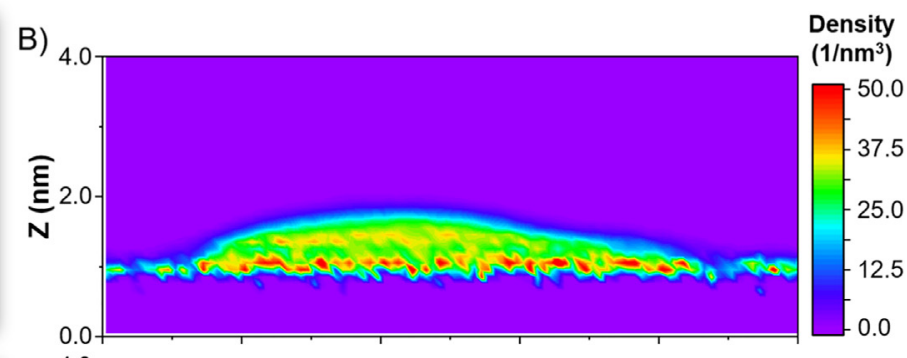

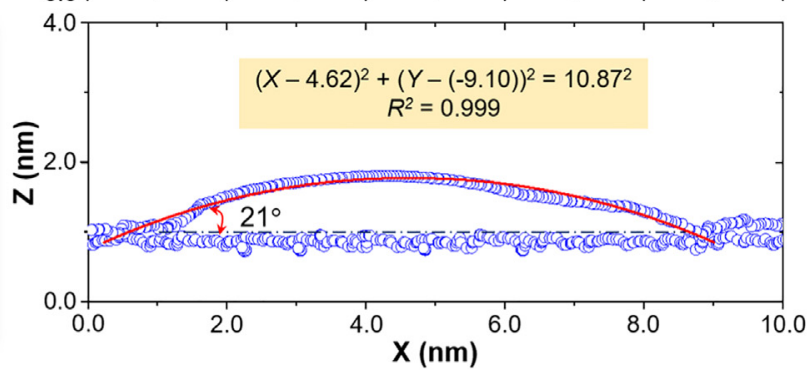

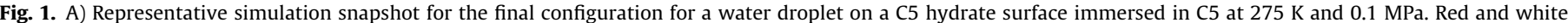

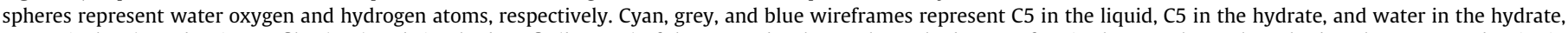

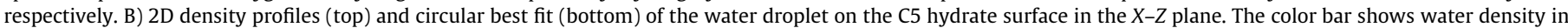

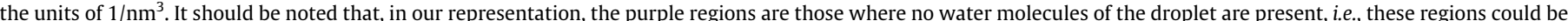

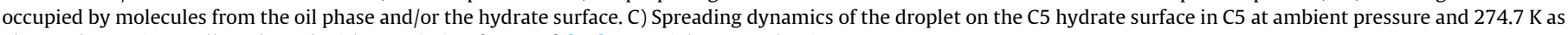
observed experimentally. Adapted with permission from Ref. [25]. Copyright 2021 Elsevier B.V.

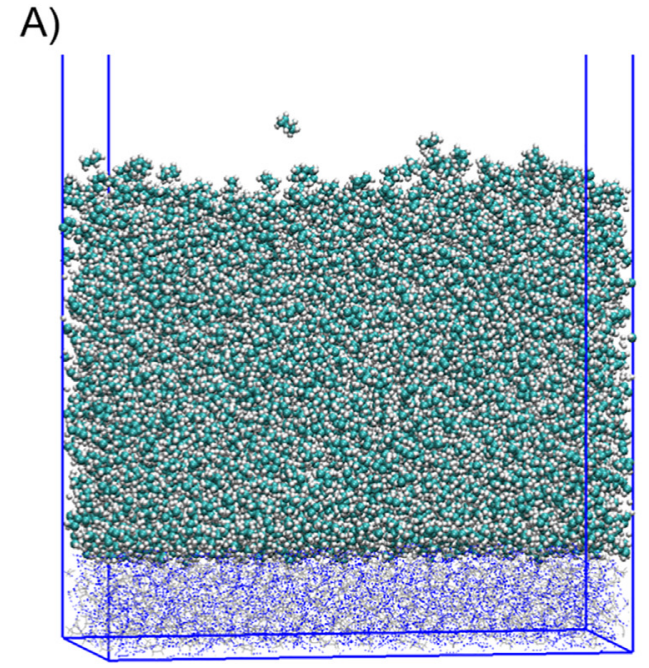

C)

\begin{tabular}{|c|c|c|c|c|c|}
\hline $\begin{array}{c}\text { Simulation } \\
\text { GROMACS-LS }\end{array}$ & $\begin{array}{c}\text { Surface tension } \\
(\mathrm{mN} / \mathrm{m})\end{array}$ & Contact Angle & Experiments & $\begin{array}{c}\text { Surface tension } \\
(\mathrm{mN} / \mathrm{m})\end{array}$ & Contact Angle \\
\hline $\mathrm{C} 5 \mathrm{Hyd}-\mathrm{C} 5$ & $42.8 \pm 2.6$ & $\cos \theta=\frac{\gamma_{s f 2}-\gamma_{s f 1}}{\gamma_{f 1,2}}$ & $\mathrm{C} 5 \mathrm{Hyd}-\mathrm{C} 5$ & $47 \pm 5$ & $\cos \theta=\frac{\gamma_{s f 2}-\gamma_{s f 1}}{\gamma_{f 1,2}}$ \\
\hline $\mathrm{C} 5 \mathrm{Hyd}-\mathrm{H}_{2} \mathrm{O}$ & $0.24 \pm 0.16$ & $\mathrm{C} 5 \mathrm{Hyd}-\mathrm{H}_{2} \mathrm{O}$ & $0.32 \pm 0.05$ & $\rightarrow \theta=23.8^{\circ}$ \\
\hline $\mathrm{C} 5-\mathrm{H}_{2} \mathrm{O}$ & $46.0 \pm 2.4$ & $\rightarrow \theta=22.3^{\circ} \pm 1.3^{\circ}$ & $\mathrm{C} 5-\mathrm{H}_{2} \mathrm{O}$ & 51 & $\rightarrow \theta$ \\
\hline
\end{tabular}

B)

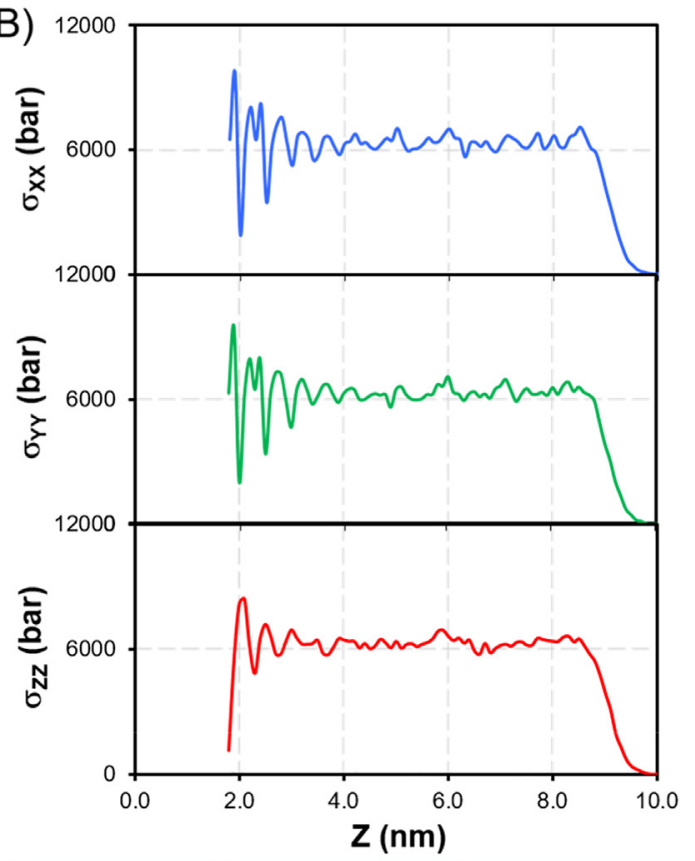

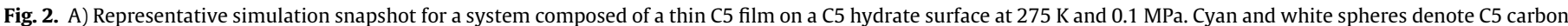

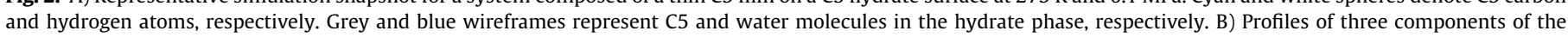

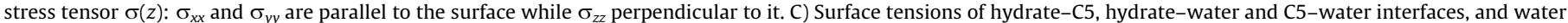
contact angle on the C5 hydrate surface in C5 calculated using Young's equation using data from the present simulations (left) and from experiments (right) [60]. 
variety of other systems [30,34-36]. The total number of atoms in the systems ranged from 15000 to 25590 .

Force Fields. Water was characterized by the TIP4P/Ice model [37], which has been used successfully in studying hydrate systems $[18,38,39]$. Methane, ethane, and $n$-dodecane were represented by employing the united-atom version of the TraPPE-UA force field [40], which correctly describes the critical properties and the vapor-liquid coexistence of linear alkanes far from the critical point. Cyclopentane (C5) and toluene (C7) were modelled implementing the General Amber Force Field (GAFF), often employed to study cyclic, organic, and pharmaceutical compounds containing $\mathrm{H}, \mathrm{C}, \mathrm{N}, \mathrm{O}, \mathrm{S}, \mathrm{P}$, and halogens [41,42]. Previous computational studies suggest that the combination of GAFF force field for modelling cyclic and organic molecules, the TIP4P/Ice model for describing water, and TraPPE-UA force field for simulating linear alkanes yields excellent agreement against experimental studies [18,19]. All non-bonded interactions were described via both electrostatic and dispersion forces using the Coulombic and 12-6 LennardJones (LJ) potentials, respectively, with the cutoff distance of 14 A. We utilized the particle-particle particle-mesh (PPPM) approach for the treatment of long-range corrections [43] and the Lorentz - Berthelot combining rules to quantify unlike LJ interactions [44].

Algorithms. Equilibrium MD simulations were performed employing the GROMACS package [45], version 2016.3. To quantify contact angles on hydrates, we first performed simulations in the NVT canonical ensemble (constant volume, temperature and number of particles) for 1 ns to relax the initial configurations, while the hydrate layer was kept rigid, and then the simulations were carried out within the NPT ensemble $(T=275 \mathrm{~K}$ and $P=0.1 \mathrm{MPa}$ for water droplet on C5 hydrate in C5 as well as C5-C7 and $T=274 \mathrm{~K}$ and $P=3.45 \mathrm{MPa}$ for water droplet on $\mathrm{C} 1-\mathrm{C} 2$ hydrate in C12) implementing the Nose-Hoover thermostat and the Berendsen/Parrinello-Rahman barostat [45]. We applied the pressure coupling algorithm only along the $Z$ direction, which allows $X$ and $Y$ dimensions of the simulation box to be kept constant.

For solid-liquid interfacial free energy calculations, all simulations were performed only within the NVT canonical ensemble while the simulations for the estimation of liquid-liquid interfacial tensions were conducted in the NVT and subsequently NPT ensembles.

Using the leapfrog algorithm the equations of motion were solved with the time step of 1.0 fs [45]. We implemented a harmonic restraint force constant of $2000 \mathrm{~kJ} / \mathrm{mol} / \mathrm{nm}$ on water, C5 as well as $\mathrm{C} 1-\mathrm{C} 2$ molecules in the hydrate phase to tether them to their initial positions [45] while other molecules in the system move freely. We conducted each NPT simulation for $\geq 150$ ns until both fluids appeared to be stable, and the droplet shape remained unchanged within a simulation time interval of $20 \mathrm{~ns}$.

\section{Results and discussion}

Wettability of Cyclopentane Hydrates immersed in Cyclopentane. We extracted contact angles from 2D density profiles obtained for the simulated water droplets (see Fig. 1B, top). The isodensity contours at water density $\rho_{\mathrm{o}}$, obtained as halfway between the water density in the hydrocarbon phase and the water bulk density [31] were used to determine the contact angle for all systems considered. Once the droplet contours were identified following the procedure described in previous studies $[31,46]$, we fit them with a circular function (see Fig. 1B, bottom). The droplet base was identified at the second hydration layer away from the hydrate surface, and the slopes of the tangent lines on both sides of the droplet were determined to calculate the contact angle.
Our simulation results yield contact angles of $\sim 21^{\circ}$ on the C5 hydrate at $275 \mathrm{~K}$ and $0.1 \mathrm{MPa}$, which is in good agreement with macroscopic experimental data reported by Thomas et al. [25] $\left(18.3-23.1^{\circ}\right)$ under similar $T$ and $P$ conditions (see Fig. 1C). These results suggest the C5 hydrate is remarkably hydrophilic, as expected, as it is largely made of water and it is structurally similar to ice. We also conducted additional simulations for a system in which the length of hydrate slab in the $X$ direction was enlarged to $L_{x}=15.579 \mathrm{~nm}$ to verify that periodic boundary conditions did not significantly affect the results presented. The water contact angle found on the enlarged hydrate surface was similar to the one obtained on the smaller one, suggesting that the systems are large enough to minimize the effect of periodic boundary conditions on the water contact angle. It should be noted that the contact angle of a simulated spherical nanodroplet [47] or of a cylindrical droplet [48] depends on the droplet size. Based on our experience, the system size used here is sufficiently large to minimize system-size effects on the estimated contact angles [30,31]. The simulation results also depend on the determination of the position and geometry of the water-liquid and water-solid interfaces, which is somewhat arbitrary. Therefore, these calculations are subject to some uncertainty, especially for highly hydrophilic surfaces [49].

As an alternative, which in some cases could yield more reliable results, one could calculate the contact angle directly from Young's equation:

$\cos \theta=\left(\gamma_{s f 2}-\gamma_{s f 1}\right) / \gamma_{f 1,2}$

which relates the contact angle to the surface free energies of solid-liquid $\left(\gamma_{s f 2}\right.$ and $\left.\gamma_{s f 1}\right)$ and liquid-liquid $\left(\gamma_{f 1,2}\right)$ interfaces $[23,50]$.

Simulated Interfacial Tensions. Molecular modelling has been shown promising in investigations of interfacial forces at solid-liquid interfaces and in fact, it has been widely employed to estimate surface free energies [51-54]. In recent years, multiple approaches have been proposed to quantify solid-liquid interfacial free energy. These methods can be categorized into either thermodynamic or mechanical routes. The thermodynamic approaches, which provide accurate results, require large sets of simulations at a huge computational cost [51]. Alternatively, the surface free energy can be determined from the mechanical approach, where it is extracted from the interfacial stress anisotropy [51]. Kirkwood and Buff [55] were the first to describe the stress tensor components against the intermolecular forces, a method which is broadly used to calculate liquid-liquid interfacial tensions [56,57]. This approach, however, is challenging when applied to solid-liquid systems [53,54], because of the anisotropy of the solid surface, which yields an alternative interpretation of the stress tensor and the surface free energy in accordance with the Shuttleworth relationship [58], applicable only when the transverse stresses have no effects on the interface. Because the hydrate surface is kept rigid in our work, such limitations can be overcome. Hence, because of its effectiveness in comparison to the thermodynamic one, in this study we employ the mechanical approach following the Irving-Kirkwood formalism [59]. This method presents a local definition of the stress tensor when velocities and interaction forces of all atoms are known.

For a system built with isotropic and planar symmetrical surfaces, such as a flat hydrate substrate exposed to water/liquid hydrocarbon, the stress tensor $\sigma(z)$ can be described with its three components: $\sigma_{z z}$ is perpendicular to the surface, and $\sigma_{x x}$ and $\sigma_{y y}$ are parallel to it. To quantify the local $\sigma(z)$, we employed the GROMACS-LS [61-63] software, which invokes the IrvingKirkwood-Noll theory for the treatment of constraints [61]. In GROMACS-LS, a cutoff radius of $2.0-2.2 \mathrm{~nm}$ is used for computing 
stresses at each frame, as it has been suggested that the stress profiles show little difference beyond this cutoff distance [61]. To compute normal and tangential stresses using GROMACS-LS, we divide the simulation box into multiple rectangular grids with cell size $L_{\text {grid }}=0.1 \mathrm{~nm}$ in three directions and calculate the averaged $\sigma$ $(z)$ over each grid cell. In Fig. 2B, we report the three components of stress tensor profiles $\sigma_{x x}(z)$ (top), $\sigma_{y y}(z)$ (middle), and $\sigma_{z z}(z)$ (bottom), which arise from the local interaction forces exerting on the liquid C5 molecules in the normal direction of the C5 hydrate surface at $275 \mathrm{~K}$ and $0.1 \mathrm{MPa}$.

Considering the stress tensor profile along the $Z$ direction perpendicular to the hydrate surface, we describe $P_{N}(z)=-\sigma_{z z}(z)$, corresponding to the normal pressure along the $Z$ direction, while $P_{T}(z)=-\frac{1}{2}\left(\sigma_{x x}(z)+\sigma_{y y}(z)\right)$, which is the tangential pressure. Using these quantities, we obtain the surface tension $\gamma_{s l}$ through the computation of the stress tensor profile [59]:

$\gamma_{s l}=\int_{0}^{L}\left(P_{N}(z)-P_{T}(z)\right) d z$

In Fig. 2C, left panel, the results of surface tensions of hydrateliquid and liquid-liquid interfaces are shown. We estimated the surface free energies of hydrate-C5 and hydrate-water interfaces as $42.8 \pm 2.6$ and $0.24 \pm 0.16 \mathrm{mN} / \mathrm{m}$, respectively. The simulated hydrate-C5 interfacial free energy is remarkably consistent with the estimates of Aman et al. [60] (47 $\pm 5 \mathrm{mN} / \mathrm{m})$, who employed a revised cohesive force model combined with experimental data obtained from micromechanical force measurements (MMF) between $\mathrm{C} 5$ hydrate particles. Our simulation results also compare well with an approximation based on wetting angle data proposed by Kwok and Neumann [64,65], who reported a value of $45 \mathrm{mN} / \mathrm{m}$. It is worth pointing out that these hydrate-C5 interfacial free energy values are comparable to the $\mathrm{C} 5$-water interfacial tension data $(46.0 \pm 2.4 \mathrm{mN} / \mathrm{m}$ obtained from the simulation results presented here and $\sim 51 \mathrm{mN} / \mathrm{m}$ from literature experiments [60]) under similar $T$ and $P$ conditions. This agreement suggests that the hydrate surface in contact with a liquid hydrocarbon may exhibit similar energetic properties as a water surface. Aman et al. [60] also reported a hydrate-water interfacial free energy of $0.32 \pm 0.05$
$\mathrm{mN} / \mathrm{m}$ (see Fig. 2C, right), which is close to our findings ( $0.24 \pm 0.16$ $\mathrm{mN} / \mathrm{m}$ ). For completeness, we point out that this estimate for the surface tension of hydrate-water interface is two orders of magnitude less than some literature data, in the range of $14-45 \mathrm{mN} / \mathrm{m}$, reported for methane, ethane, and propane hydrates [66-68]. However, these estimates were indirectly determined from porous media measurements, where huge discrepancies in wetting angle and pore size may lead to uncertainties [69].

Applying Young's equation [Eq. (1)] with the values of computational and experimental surface free energies shown in Fig. 2 yields the equilibrium contact angles of $22.3^{\circ}$ and $23.8^{\circ}$, respectively, for the droplet on the C5 hydrate surface immersed in C5. These values are in excellent agreement with the data obtained directly from our simulations (see Fig. 1B, top), as well as from recent experimental observations [25] (see Fig. 1C).

Wettability of Methane-Ethane Hydrates Immersed in nDodecane. Most of the wetting studies on clathrate hydrates were conducted on hydrates stable at atmospheric pressure, i.e., using C5 hydrates [16,25], even though realistic flow assurance applications typically encounter high $P$ and low $T$ (i.e., $>2 \mathrm{MPa}$ and $<277 \mathrm{~K}$ ) in liquid-hydrocarbon-dominated phases [70]. To examine somewhat realistic conditions, we investigate the wetting properties of C1-C2 hydrates immersed in bulk C12 at $274 \mathrm{~K}$ and 3.45 MPa (see Fig. 3A). After analysing 2D density profiles obtained for the simulated water droplets (Fig. $3 \mathbf{B}$, top), we determined the contact angles (Fig. 3B, bottom). Our simulations yield a contact angle of $34^{\circ}$ on C1-C2 hydrates immersed in C12, which agrees well with the simulated data reported by Naullage et al. [71] $\left(34 \pm 2^{\circ}\right)$, who considered sI methane hydrates at slightly higher $T(277 \mathrm{~K})$ and $P(10 \mathrm{MPa})$ conditions. We also simulated the water contact angle on C1-C2 hydrates immersed in C12 under atmospheric pressure. The result $\left(\sim 30^{\circ}\right)$ is comparable to data obtained at higher pressures, suggesting that moderate changes in pressures up to $\sim 20 \mathrm{MPa}$ only marginally impact the wetting properties of hydrate surfaces, a result which is consistent with data from other studies [72,73]. Our results suggest that, similarly to the C5 hydrate, the C1-C2 hydrate is also highly hydrophilic. For completeness, it should be mentioned that Molinero et al. [74] showed that wetting a clathrate hydrate surface with a thin water film
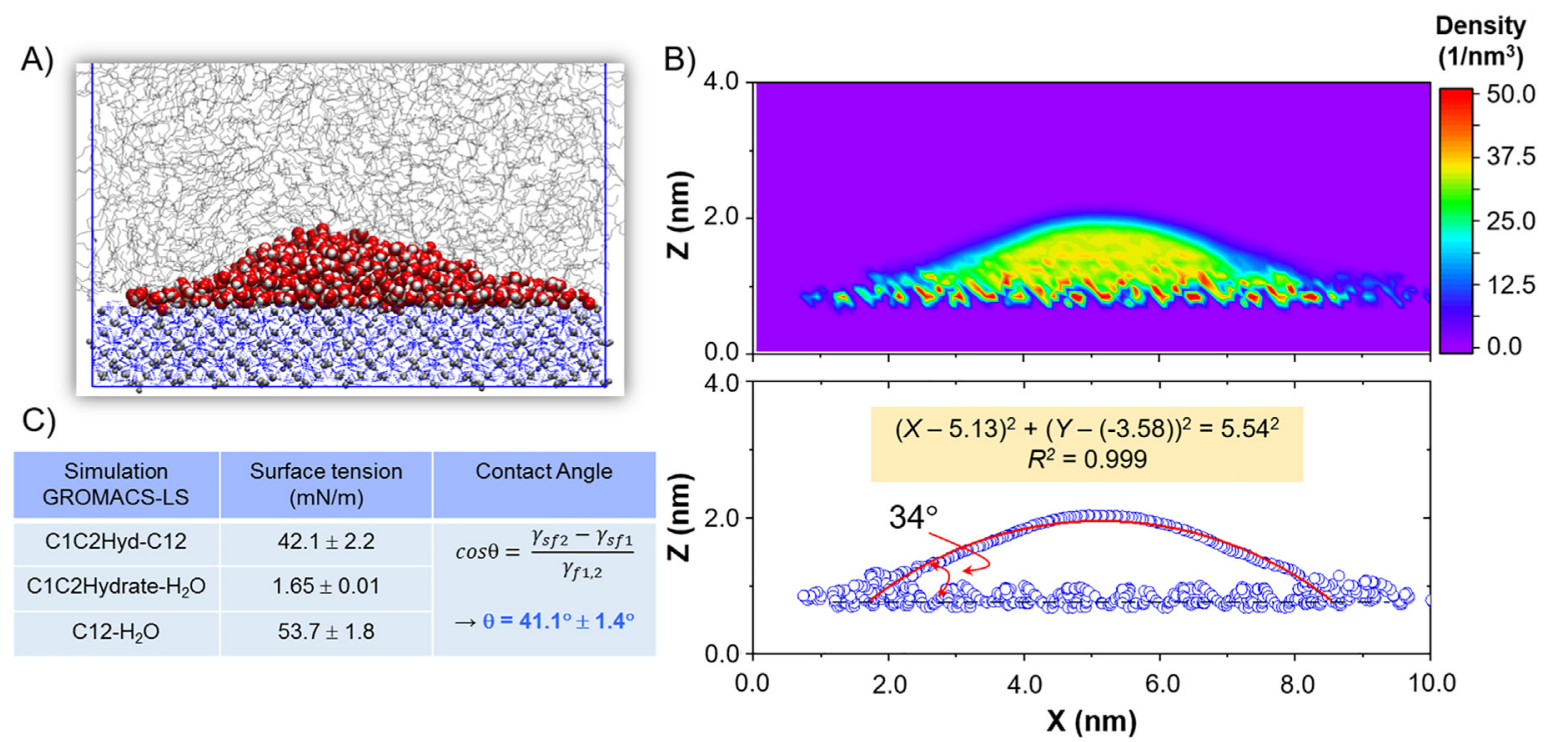

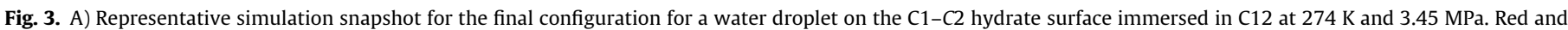

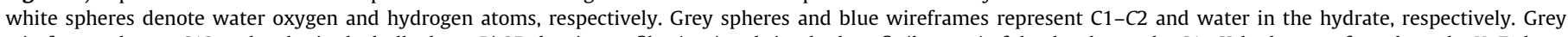

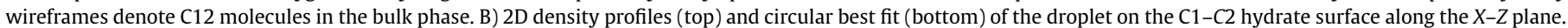

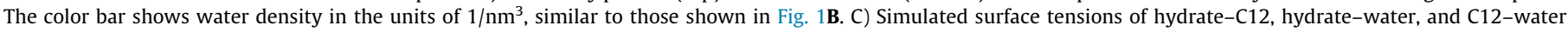
interfaces, as well as the corresponding water contact angle on the C1-C2 hydrate in C12 as calculated using Young's equation from the surface tension data. 
could lead to strong adsorption of alkanes, suggesting that the resultant interface is oleophilic.

Using Young's equation [Eq. (1)] with the simulated water contact angle of $34 \pm 2^{\circ}$, the hydrate-water interfacial free energy of $33 \pm 4 \mathrm{mN} / \mathrm{m}[67,75]$, and the experimental C12-water interfacial tension of $53 \mathrm{mN} / \mathrm{m}$ [76], Naullage et al. [71] reported the hydrate-C12 interfacial free energy of $76.9 \pm 2 \mathrm{mN} / \mathrm{m}$. Contrarily, we estimated the surface tensions of hydrate-C12 and hydratewater interfaces at $42.1 \pm 2.2$ and $1.65 \pm 0.01 \mathrm{mN} / \mathrm{m}$, respectively, directly using GROMACS-LS [61-63] (see Fig. 3C). Our calculations yield similar values for the hydrate-C12 $(42.1 \pm 2.2 \mathrm{mN} / \mathrm{m})$ and hydrate-C5 $(42.8 \pm 2.6 \mathrm{mN} / \mathrm{m})$ interfacial free energies at similar $T(274-275 \mathrm{~K})$. Our simulation results for the surface free energy of the C1-C2 hydrate-water interface $(1.65 \pm 0.01 \mathrm{mN} / \mathrm{m})$ obtained at $274 \mathrm{~K}$ are comparable to those for the $\mathrm{C} 5$ hydrate-water interface $(0.32 \pm 0.05 \mathrm{mN} / \mathrm{m})$ at $275 \mathrm{~K}$ (see Fig. $2 \mathbf{C}$, left). These values are, however, much lower than those used by Naullage et al. $(33 \pm 4 \mathrm{mN} / \mathrm{m})$ [71], which were obtained by determining equilibrium temperatures of hydrate in hydrate-filled porous media and then employing the Gibbs-Thompson equation to estimate the surface free energy for the hydrate-water interface (using 4 $100 \mathrm{~nm}$ pore diameters with an assumed hydrate wetting angle of $0^{\circ}$ ) [60]. Employing GROMACS-LS [61-63], we obtain values for the simulated C12-water interfacial tension $(53.7 \pm 1.8 \mathrm{mN} /$ $\mathrm{m})$ that are remarkably consistent with experimental data (53 $\mathrm{mN} / \mathrm{m}$ ) [76]. Applying Young's equation using these values yields equilibrium contact angles of $\sim 41^{\circ}$ for a water droplet on C1-C2 hydrates immersed in $\mathrm{C} 12$, which is in excellent agreement with the values obtained directly from our simulations (see Fig. 3B, top). We conclude that the value for the $\mathrm{C} 1$ hydrate-C12 surface free energy found by Naullage et al. [71] $(76.9 \pm 2.0 \mathrm{mN} / \mathrm{m})$ is unrealistically high, compared to our estimates, likely as a consequence of the approximations invoked.

Although our simulation results agree quantitatively with the contact angle measurements reported by Thomas et al. [25], they do not agree with Brown et al. [16], who reported water contact angles of $94.2^{\circ} \pm 8.5^{\circ}$ on a C5 hydrate immersed in C5. Very recently, Stoner et al. [28] suggested that surface abnormalities as well as hydrate film growth may slow the spreading of a water droplet deposited on the hydrate surface, thereby affecting the observed contact angle (see Fig. 4A). We hypothesize that surface heterogeneities, such as roughness (at various length scales), could affect the measured contact angle, particularly noting that the hydrate surfaces used by Thomas et al. were very smooth, rigid, and flat [25], and noting that Young's equation (see Figs. 2 and 3 ), is only applicable to smooth and flat surfaces [23,50]. Our hypothesis is supported by the experimental observations by Stoner et al. [28], and also by Spencer et al. [77], who quantified the effect of heterogeneity on contact line pinning. Factors considered included chemical heterogeneity, surface roughness, and interfacial wetting states, which possibly create contact line pinning $[78,79]$. These authors conducted experiments to measure water contact angles on various rough surfaces with precisely controlled surface chemistry, showing that pinning can shift the contact angles on hydrophilic rough surfaces to more hydrophobic values (reported in detail in ref. [77]). Note that pinning effects induced by roughness become less significant under conditions of strong capillary forces that promote complete wetting [77].

Pinning Effects on Cyclopentane Hydrates. Employing MD, we examine the impact of contact line pinning on the wetting properties for C5 hydrate surfaces immersed in C5. First, we generated rough hydrate surfaces (see Fig. $4 \mathbf{B}$, left) through hydrate growth from water droplets immersed in liquid hydrocarbons spread on flat hydrates (see Fig. 1A/3A for the various configurations). We then performed simulations for water droplets of different sizes on the produced atomically rough C5 hydrate surfaces at $275 \mathrm{~K}$ and 0.1 MPa (see Fig. 4B, right). In Fig. 4C, we present 2D density profiles obtained for water droplets of various sizes on the rough C5 hydrate. Extracting the droplet contours and further fitting them with a circular function, we obtained contact angle values (see Fig. 4D). Analysis of the simulation results allows us to distinguish two contact angles for each droplet, i.e., the 'local' and the 'apparent' contact angles. Note that the local contact angle, as defined here, has been referred to as 'intrinsic' contact angle in the literature [81]. On real surfaces, chemically heterogeneous and geometrically rough [82-84], these different contact angles could be characterized [85], which can be difficult to assess experimentally. In our nomenclature, we also distinguish local and apparent contact angle from the 'Young' contact angle, which would correspond to the contact angle obtained in an atomically flat and chemically homogeneous substrate. On a rough surface, the local contact angle is formed between the tangent to the local solid surface and the tangent to liquid-liquid interface. The apparent contact angle is instead formed by the macroscopicgeometrical solid surface and the tangent to liquid-liquid interface (see Figure S2 of the SI) [85]. On an atomically smooth and chemically homogeneous surface, in the absence of pinning phenomena, the apparent contact angle is expected to correspond to the local and to the Young contact angles [86]. Following Shuttleworth and Bailey [87] (see Figure S2), we propose that the local angle is the sum of the apparent angle and the angle of the surface slope at the liquid-solid contact point [81].

Within the size limits accessible to our simulations, we observe that the effect of the contact line pinning created by surface heterogeneity becomes insignificant when the droplet volume increases from 28 to $68 \mathrm{~nm}^{3}$. This is probably because the base radius of the droplet increases above the length scale controlled by the local roughness of the surfaces considered in our simulations (see Figure S3 of the SI). After a certain droplet size $\left(68 \mathrm{~nm}^{3}\right)$, we observe a sharp increase in the observed contact angle, which is likely due to pronounced contact line pinning effects, which maintain the constant base of the droplet despite the increased droplet volume, yielding a local contact angle as high as $\sim 75^{\circ}$, which is comparable to the experimental data reported by Brown et al. [16] $\left(94.2^{\circ} \pm 8.5^{\circ}\right)$ and by Stoner et al. [28] $\left(94^{\circ} \pm\right.$ $17^{\circ}$ ). Although the length scales of the two approaches are different, we find this agreement encouraging. Note that the estimated characteristic length of the rough feature (defined as the ratio of the height of roughness to the base radius of the droplet) obtained from experiments $(\sim 0.083$, estimated from Fig. $4 \mathbf{A})$ and this simulation study $(0.085-0.096)$ are comparable, justifying the credibility of our comparison.

Pinning Effects on Methane-Ethane Hydrates. We also investigate the influence of contact line pinning on wetting properties on rough $\mathrm{C} 1-\mathrm{C} 2$ hydrates immersed in $\mathrm{C} 12$ at $274 \mathrm{~K}$ and 3.45 MPa (see Fig. 5A). We implemented simulation and analysis procedures similar to those employed to study water droplets on C5 hydrates. In Fig. 5B, the results of 2D density profiles for the simulated water droplets of various sizes on the rough $\mathrm{C} 1-\mathrm{C} 2$ hydrate are shown. We obtained local and apparent contact angles (see Fig. 5C) by fitting the extracted droplet contours using a circular function. In contrast to the results shown for water droplets on C5 hydrates (see Fig. 4D), the local/apparent contact angles on rough $\mathrm{C} 1-\mathrm{C} 2$ hydrates immersed in C12 increase significantly (from $30^{\circ}$ to $45^{\circ}$ ) when the droplet volume increases from 28 to $42 \mathrm{~nm}^{3}$. The pinning effects appear to be more pronounced for small droplets on rough $\mathrm{C} 1-\mathrm{C} 2$ hydrates in C12 compared to the ones on rough C5 hydrates (see Figure S3). This difference could be attributed to the lower water wettability of the $\mathrm{C} 1-\mathrm{C} 2$ hydrates in $\mathrm{C} 12\left(\theta_{C 1-C 2 i n C 12}\left(34-41^{\circ}\right)>\theta_{C \operatorname{CinC5}}\left(21-23^{\circ}\right)\right.$, see Figs. 1,2 and 3$)$. Recently, when studying the variation of contact angle due to pinning forces, Ozcelik et al. [80] observed that pinning effects at a 
A)
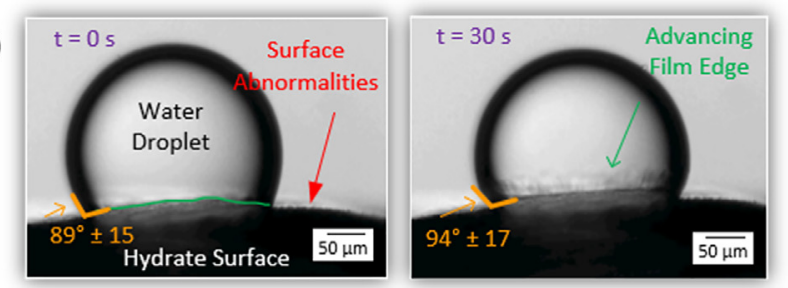

B)
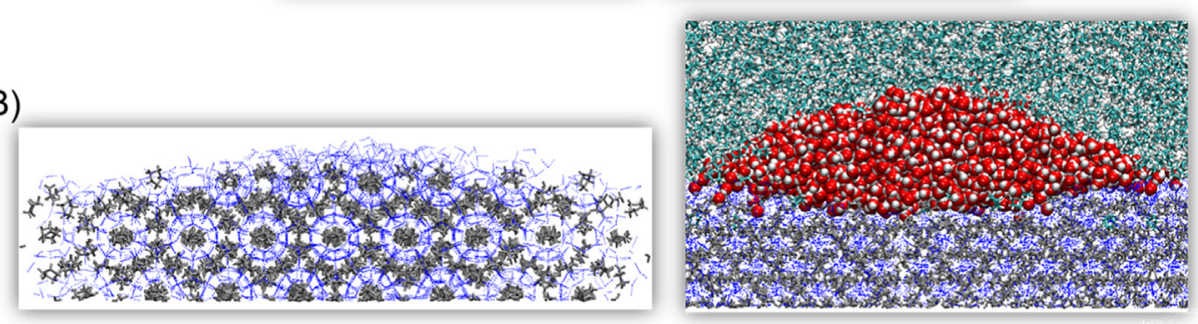

\section{Density}

$\left(1 / \mathrm{nm}^{3}\right)$
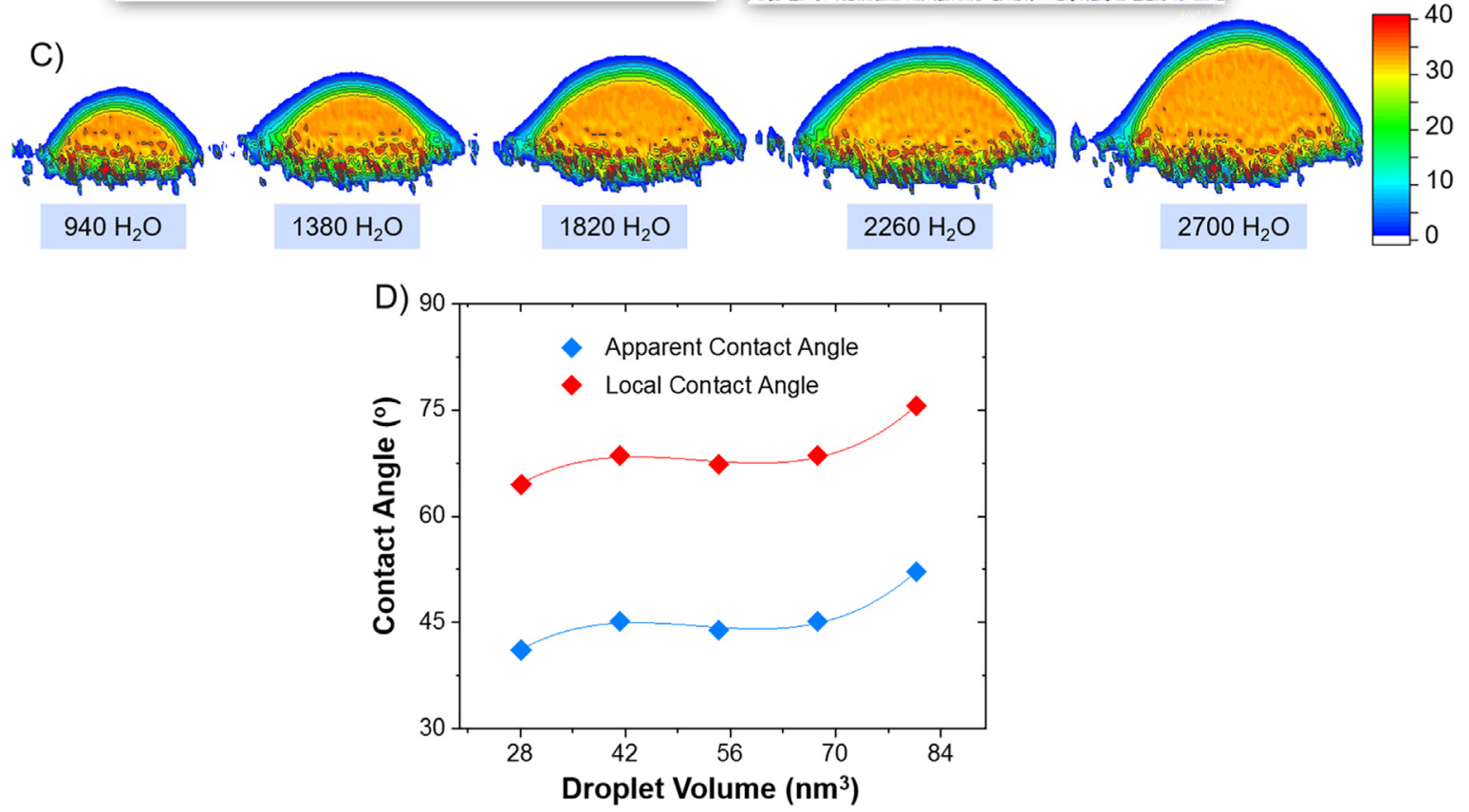

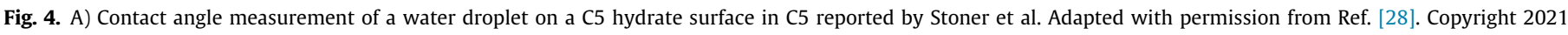

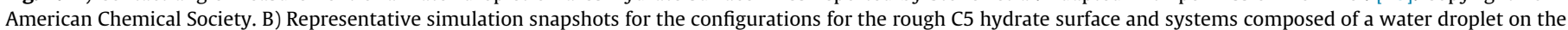

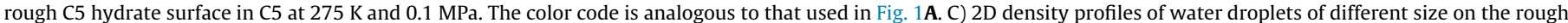

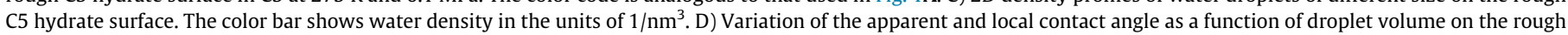

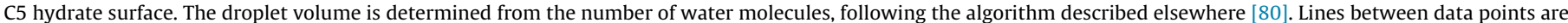

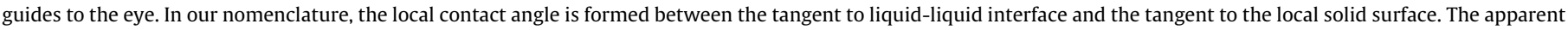
contact angle is instead formed by the tangent to the liquid-liquid interface and the macroscopic-geometrical solid surface.

microscopic level become predominant with the increase of surface heterogeneity and lower surface wettability. When the droplet volume continues to increase, the contact angle increases slightly on rough $\mathrm{C} 1-\mathrm{C} 2$ hydrates, leading to local contact angles of $\sim 78^{\circ}$, consistent with those measured experimentally in the high-pressure MMF apparatus $\left(85^{\circ} \pm 5^{\circ}\right)[88]$ under similar conditions (Fig. 5D).

Contact-line Pinning Force. The results in Figs. 4 and 5 are consistent with the observation that a pinned contact line maintains the base of the droplet constant as the droplet volume increases, which ultimately yields an increase in the measured contact angle. The so-called pinning force is responsible for an energy barrier that the contact line must overcome to move from one metastable state to another [80]. To quantify the pinning effects, we calculate the pinning force $F_{p i n}$ on the three-phase contact line using the following expression $[80,89,90]$ :

$F_{\text {pin }}=\gamma_{f 1,2}\left(\cos \theta_{\infty}-\cos \theta\right)$
In Eq. (3), $\gamma_{f 1,2}$ is the water-hydrocarbon interfacial tension, $\theta_{\infty}$ is the contact angle on an atomically flat and chemically homogeneous surface without contact line pinning ('Young' contact angle) and $\theta$ is the local contact angle measured from simulations (or experiments).

In Fig. 6, left, we show the results of pinning force as a function of the droplet volume on the rough C5 hydrates immersed in C5 (blue) at $275 \mathrm{~K}$ and $0.1 \mathrm{MPa}$ and on the rough $\mathrm{C} 1-\mathrm{C} 2$ hydrates immersed in $\mathrm{C} 12$ (red) at $274 \mathrm{~K}$ and $3.45 \mathrm{MPa}$. We observe that the pinning force on the contact line of water-C5-C5 hydrate barely changes for droplet volumes up to $\sim 70 \mathrm{~nm}^{3}$ but quickly grows when further increasing the droplet volume, while the pinning force on the water-C12-C1-C2 hydrate contact line initially increases rapidly, and then grows only slightly when the droplet volume continues to increase. This suggests that the interactions between droplet fluid and the surrounding liquid strongly influence the relation between pinning force and droplet size when (as in our simulations) the rough surface morphologies are similar. 
A)
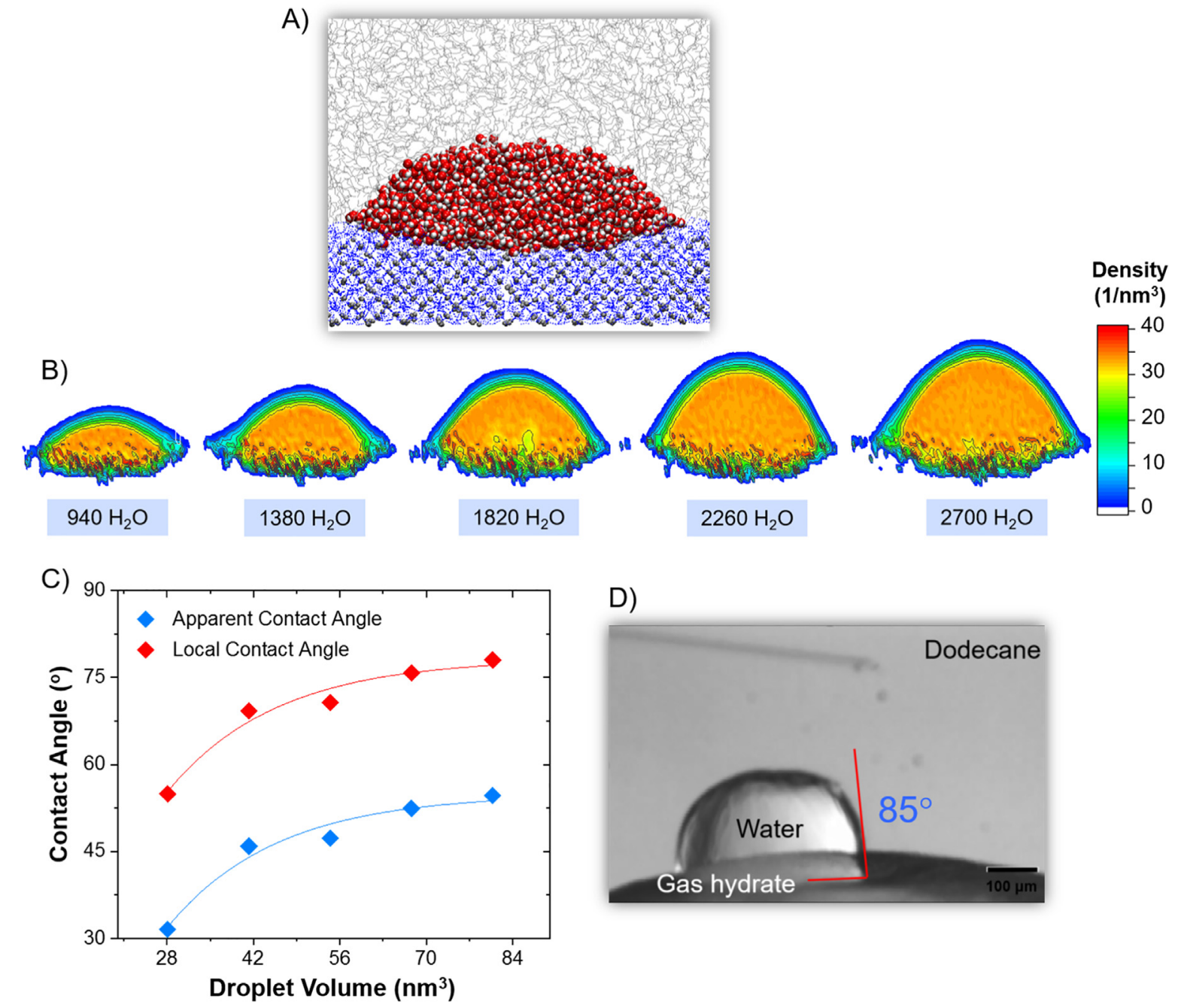

D)

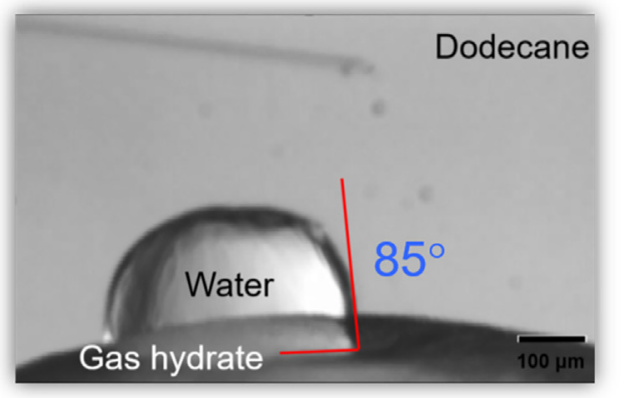

Droplet Volume (nm $\left.{ }^{3}\right)$

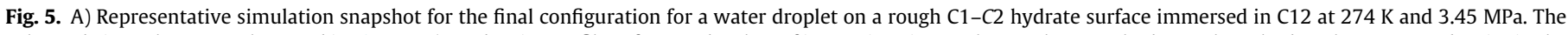

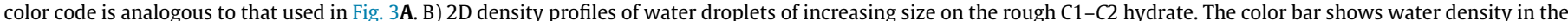

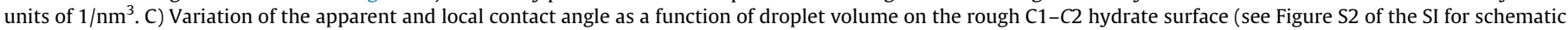

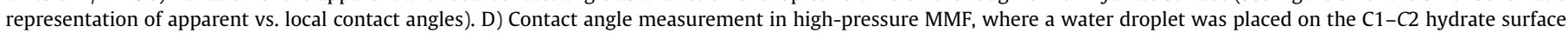
immersed in C12 at $274 \mathrm{~K}$ and $3.45 \mathrm{MPa}$, reported by Hu. Adapted with permission from Ref. [88].
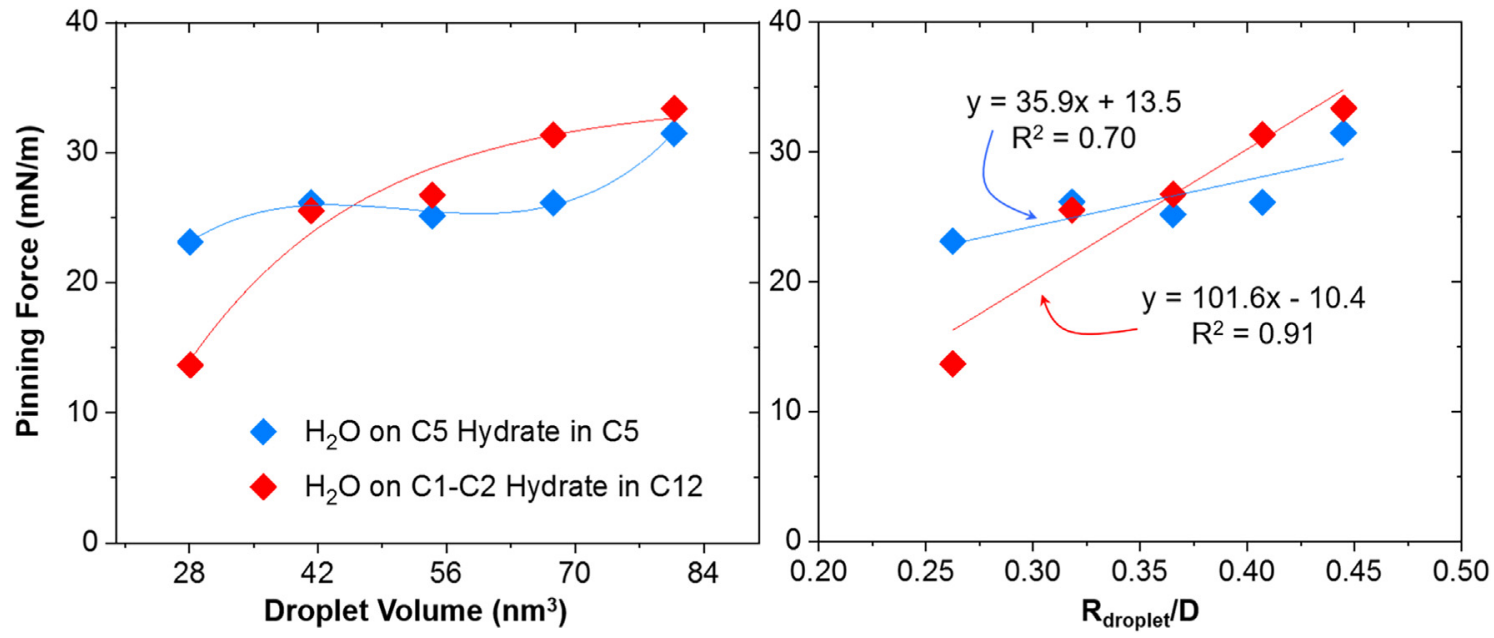

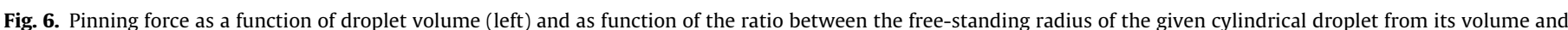

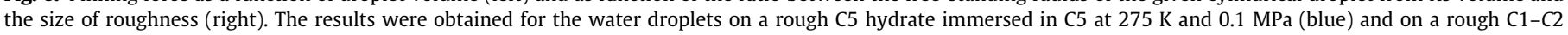
hydrate immersed in $\mathrm{C} 12$ at $274 \mathrm{~K}$ and $3.45 \mathrm{MPa}$ (red). Lines are guides to the eye. 
We notice that the pinning forces obtained for both simulated systems are comparable when the droplet volume reaches $\sim 82 \mathrm{~nm}^{3}$. When the droplet volume increases further, eventually the droplets completely wet the rough hydrate surfaces due to the significant increase of capillary force to values much greater than the pinning force $[77,91]$. It is expected that droplets that are much smaller than the roughness length scale would fully wet the hydrate surfaces between two pinning spots, yielding water contact angles similar to those found on flat hydrates. It is perhaps interesting to point out that our results show a linear correlation between the pinning force and the ratio between the freestanding radius of the cylindrical droplet (independent of the droplet's wetting shape) and the extent of roughness (estimated as the distance between two pinning points) for both rough C5 and C1-C2 hydrate surfaces (see Fig. 6, right). This linear correlation is consistent with previous studies $[80,90]$. It should be noted that the ratio between the free-standing radius of the cylindrical droplet and the extent of roughness eventually yields a parameter that could be used to tune the pinning force through the design of appropriate surface features [90].

Work of Adhesion. One of the most useful quantities to characterize wetting properties for a solid surface is the work of adhesion, often defined for a liquid droplet on a solid substrate, $W_{12 s} . W_{12 S}$ is described as the energy per unit area required to separate the droplet from the solid. It is determined from the Young contact angle $\theta_{\infty}$ and water-hydrocarbon interfacial tension $\gamma_{f 1,2}$ using the Young-Dupré equation [92]:

$W_{12 S}=\gamma_{f 1,2}\left(1+\cos \theta_{\infty}\right)$

With the known values of the water Young contact angle on C5 hydrates immersed in $\mathrm{C} 5\left(22.3^{\circ}\right)$ and the $\mathrm{C} 5$-water interfacial tension $(46.0 \pm 2.4 \mathrm{mN} / \mathrm{m})$, the work of adhesion was found to be $\sim 88.6 \mathrm{mN} / \mathrm{m}$. Similarly, the work of adhesion for the water droplet on the C1-C2 hydrate surfaces immersed in C12 was found to be $\sim 98.2 \mathrm{mN} / \mathrm{m}$ using the water contact angle and interfacial tension for $\mathrm{C} 12$ systems (see Fig. 3B and 3C). In Fig. 7, we compare the work of adhesion calculated from our simulations (yellow columns) to experimental results. For example, Chen et al. [93] recently reported that the equilibrium attractive forces measured experimentally between a water droplet $\left(D_{d}=2.00 \pm 0.05 \mathrm{~mm}\right)$ and a C5 hydrate particle $\left(D_{h}=4.00 \pm 0.22 \mathrm{~mm}\right)$ immersed in C5

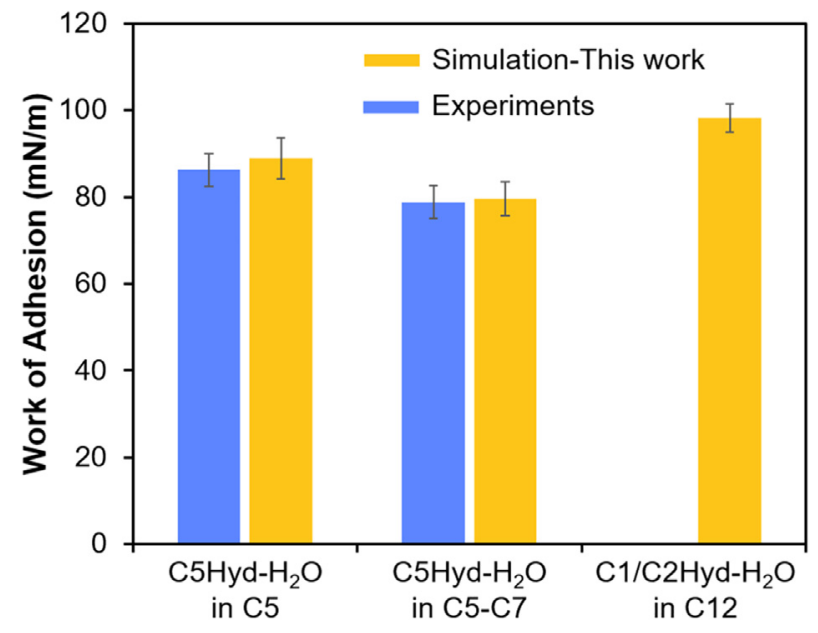

Fig. 7. Work of adhesion between a water droplet and a hydrate particle immersed in liquid hydrocarbons. The results were obtained for the systems composed of the water droplet and C5 hydrate particles in either pure C5 or C5-C7 mixture at $275 \mathrm{~K}$ and $0.1 \mathrm{MPa}$, and of the water droplet and the C1-C2 hydrate particle in $\mathrm{C} 12$ at $274 \mathrm{~K}$ and $3.45 \mathrm{MPa}$. are of $0.23 \pm 0.01 \mathrm{mN}$ at $275 \pm 0.5 \mathrm{~K}$, yielding a work of adhesion of $\sim 86.3 \mathrm{mN} / \mathrm{m}$ (see Fig. 7, blue), which is in excellent agreement with our simulation results $(88.6 \mathrm{mN} / \mathrm{m})$. To test applicability and reliability of the method, we constructed additional simulation systems composed of the droplet on C5 hydrates immersed in ( $50 \% \mathrm{C} 5+50 \% \mathrm{C} 7)$ mixtures at $275 \mathrm{~K}$. We obtained a Young contact angle of $\sim 30^{\circ}$ and an interfacial tension of $\sim 42.7 \pm 2.1 \mathrm{mN} / \mathrm{m}$ (close to the experimental value of $\sim 42.2 \mathrm{mN} / \mathrm{m}$ obtained for this interface) [94], leading to a work of adhesion of $\sim 79.7 \mathrm{mN} / \mathrm{m}$, which agrees well with experimental data reported by Chen et al. [93] $(78.8 \mathrm{mN} / \mathrm{m})$. This suggests that the direct simulation of water contact angle on an atomically smooth, chemically homogeneous hydrate surface immersed in a liquid, combined with the surface free energy estimated for water-surrounding liquid interfaces could be used to predict, rather accurately, experimental adhesion force measurements.

\section{Conclusions}

The literature shows discrepancies on contact angles measured for water on hydrate particles $[16,25,28,88]$. Such discrepancies hamper technological progress, specifically in flow assurance. We hypothesize that surface heterogeneity is responsible for the wide variation of contact angles reported. To test this hypothesis at the molecular-level, we implement an arsenal of computational materials chemistry methods. We compute directly contact angles and we estimate surface free energies of solid-liquid and liquid-liquid interfaces. The simulation results show excellent agreement with macroscopic experimental observations for both atomically smooth and rough hydrate surfaces under similar temperature and pressure conditions. We conclude that contact line pinning, prompted by surface heterogeneities such as roughness, is responsible for hindering the spreading of the water droplet on the hydrate surface, leading to contact angles larger than those expected based on thermodynamic arguments alone (i.e., the 'Young' contact angle in our nomenclature). This interpretation allows us to reconcile the discrepancy between experimental contact angle values reported as low as $20^{\circ}$, and as high as $80-90^{\circ}$ in the literature $[16,25,28,88]$. We found that our estimates for the work of adhesion are consistent with experimental measurements [93], suggesting that simulations such as those presented here could be useful for the estimation of adhesion force interactions between hydrate particles and water droplets. To our knowledge, this is the first determination of the hydrate-water adhesive forces achieved by employing molecular simulations under realistic conditions, as most previous experimental studies were performed using hydrates stable at atmospheric pressure, such as cyclopentane [93,95-97]. Our work contributes to closing the gap between experimental and realistic models of clathrate hydrates. Because understanding the wetting characteristics of hydrates will provide insights into their formation, growth, and agglomeration, our analysis will be beneficial to quantify the impact of additives on hydrate-forming systems in future studies. For example, it could be of interest to conduct a molecular level study on the effects of various additives adsorbed on hydrate surfaces on wetting properties of relevance to flow assurance.

\section{CRediT authorship contribution statement}

Anh Phan: Conceptualization, Methodology, Investigation, Visualization, Writing - original draft. Hannah M. Stoner: Writing - review \& editing. Michail Stamatakis: Funding acquisition, Writing - review \& editing. Carolyn A. Koh: Funding acquisition, Writing - review \& editing. Alberto Striolo: Funding acquisition, Writing - review \& editing. 


\section{Declaration of Competing Interest}

The authors declare that they have no known competing financial interests or personal relationships that could have appeared to influence the work reported in this paper.

\section{Acknowledgements}

Financial support was generously provided, in part, by the UK EPSRC, under grant number EP/T004282/1 (AS), and by the US National Science Foundation, under grant number CBET 2015201 (CAK, HMS). Generous allocations of computing time were provided by ARCHER2, the UK National Supercomputing Service (http://www.archer2.ac.uk) and by the University College London Research Computing Platforms Support (Young).

\section{Appendix A. Supplementary data}

Supplementary data to this article can be found online at https://doi.org/10.1016/j.jcis.2021.12.083.

\section{References}

[1] A. Striolo, A. Phan, M.R. Walsh, Molecular properties of interfaces relevant for clathrate hydrate agglomeration, Curr Opin Chem Eng 25 (2019) 57-66.

[2] F. Sicard, A. Striolo, Role of structural rigidity and collective behaviour in the molecular design of gas hydrate anti-agglomerants, Mol Syst Des Eng (2021).

[3] D. Lee, Y. Lee, J. Lim, Y. Seo, Guest enclathration and structural transition in $\mathrm{CO} 2+\mathrm{N}-2+$ methylcyclopentane hydrates and their significance for $\mathrm{CO} 2$ capture and sequestration, Chem Eng J 320 (2017) 43-49.

[4] D.Y. Koh, H. Kang, J. Jeon, Y.H. Ahn, Y. Park, H. Kim, H. Lee, Tuning Cage Dimension in Clathrate Hydrates for Hydrogen Multiple Occupancy, J Phys Chem C 118 (6) (2014) 3324-3330.

[5] S. Moon, Y.H. Ahn, H. Kim, S. Hong, D.Y. Koh, Y. Park, Secondary gaseous guestdependent structures of binary neopentyl alcohol hydrates and their tuning behavior for potential application to CO2 capture, Chem Eng J 330 (2017) 890898.

[6] S. Denning, A.A.A. Majid, J.M. Lucero, J.M. Crawford, M.A. Carreon, C.A. Koh, Metal-Organic Framework HKUST-1 Promotes Methane Hydrate Formation for Improved Gas Storage Capacity, Acs Appl Mater Inter 12 (47) (2020) 5351053518.

[7] N.I. Papadimitriou, I.N. Tsimpanogiannis, I.G. Economou, A.K. Stubos, Monte Carlo simulations of the separation of a binary gas mixture $(\mathrm{CH} 4+\mathrm{CO} 2)$ using hydrates, Phys Chem Chem Phys 20 (44) (2018) 28026-28038.

[8] A. Hassanpouryouzband, E. Joonaki, M.V. Farahani, S. Takeya, C. Ruppel, J.H. Yang, N.J. English, J.M. Schicks, K. Edlmann, H. Mehrabian, Z.M. Aman, B. Tohidi, Gas hydrates in sustainable chemistry, Chem Soc Rev 49 (15) (2020) 52255309.

[9] S.D. Seo, S.Y. Hong, A.K. Sum, K.H. Lee, J.D. Lee, B.R. Lee, Thermodynamic and kinetic analysis of gas hydrates for desalination of saturated salinity water, Chem Eng J 370 (2019) 980-987.

[10] X.J. Fu, J. Jimenez-Martinez, T.P. Nguyen, J.W. Carey, H. Viswanathan, L. CuetoFelgueroso, R. Juanes, Crustal fingering facilitates free-gas methane migration through the hydrate stability zone, P Natl Acad Sci USA 117 (50) (2020) 31660-31664.

[11] T. Bui, D. Monteiro, L. Vo, A. Striolo, Synergistic and Antagonistic Effects of Aromatics on the Agglomeration of Gas Hydrates, Sci Rep-Uk 10 (1) (2020) 111.

[12] M.A. Kelland, Production Chemicals for the Oil and Gas Industry, CRC Press, 2010.

[13] E. Dendy Sloan, C.A. Koh, Clathrate Hydrates of Natural Gases, CRC Press Taylor \& Francis Group, 2007.

[14] E. Dendy Sloan, C.A. Koh, A. Sum, Natural Gas Hydrates in Flow Assurance, Elsevier, Boston, 2011.

[15] L.E. Zerpa, J-L. Salager, C.A. Koh, E.D. Sloan, A.K. Sum, Surface Chemistry and Gas Hydrates in Flow Assurance, Ind Eng Chem Res 50 (1) (2011) 188-197.

[16] E.P. Brown, S. Hu, J. Wells, X. Wang, C.A. Koh, Direct Measurements of Contact Angles on Cyclopentane Hydrates, Energ Fuel 32 (6) (2018) 6619-6626.

[17] A. Phan, T. Bui, E. Acosta, P. Krishnamurthy, A. Striolo, Molecular mechanisms responsible for hydrate anti-agglomerant performance, Phys Chem Chem Phys 18 (36) (2016) 24859-24871.

[18] A. Phan, M. Stamatakis, C.A. Koh, A. Striolo, Correlating Antiagglomerant Performance with Gas Hydrate Cohesion, Acs Appl Mater Inter 13 (33) (2021) 40002-40012.

[19] T. Bui, A. Phan, D. Monteiro, Q. Lan, M. Ceglio, E. Acosta, P. Krishnamurthy, A. Striolo, Evidence of structure-performance relation for surfactants used as antiagglomerants for hydrate management, Langmuir 33 (9) (2017) 2263 2274
[20] F. Sicard, T. Bui, D. Monteiro, Q. Lan, M. Ceglio, C. Burress, A. Striolo, Emergent Properties of Antiagglomerant Films Control Methane Transport: Implications for Hydrate Management, Langmuir 34 (33) (2018) 9701-9710.

[21] K. Kinnari, J. Hundseid, X.Y. Li, K.M. Askvik, Hydrate Management in Practice, J Chem Eng Data 60 (2) (2015) 437-446.

[22] I. Rao, C.A. Koh, E.D. Sloan, A.K. Sum, Gas Hydrate Deposition on a Cold Surface in Water-Saturated Gas Systems, Ind Eng Chem Res 52 (18) (2013) 6262-6269.

[23] J.N. Israelachvili, Intermolecular and Surface Forces, 2nd edition ed.,., Academic Press, 2011.

[24] T. Bui, F. Sicard, D. Monteiro, Q. Lan, M. Ceglio, C. Burress, A. Striolo, Antiagglomerants Affect Gas Hydrate Growth, J Phys Chem Lett 9 (12) (2018) 3491-3496

[25] F. Thomas, D. Dalmazzone, J.F. Morris, Contact angle measurements on cyclopentane hydrates, Chem Eng Sci 229 (2021) 116022.

[26] G. Zylyftari, A. Ahuja, J.F. Morris, Nucleation of cyclopentane hydrate by ice studied by morphology and rheology, Chem Eng Sci 116 (2014) 497-507.

[27] S.-L. Li, C.-Y. Sun, B. Liu, Z.-Y. Li, G.-J. Chen, A.K. Sum, New Observations and Insights into the Morphology and Growth Kinetics of Hydrate Films, Sci RepUk 4 (1) (2014) 4129.

[28] H.M. Stoner, A. Phan, A. Striolo, C.A. Koh, Water Wettability Coupled with Hydrate Film Growth on Realistic Cyclopentane Clathrate Surfaces, Langmuir 37 (42) (2021) 12447-12456.

[29] F. Takeuchi, M. Hiratsuka, R. Ohmura, S. Alavi, A.K. Sum, K. Yasuoka, Water proton configurations in structures I, II, and $\mathrm{H}$ clathrate hydrate unit cells, J Chem Phys 138 (12) (2013) 124504.

[30] T. Thi Bao Le, C. Divine-Ayela, A. Striolo, D.R. Cole, Effects of surface contamination on the interfacial properties of $\mathrm{CO} 2 /$ water/calcite systems, Phys Chem Chem Phys 23 (2021) 18885.

[31] T.T.B. Le, A. Striolo, D.R. Cole, Supercritical CO2 Effects on Calcite Wettability: A Molecular Perspective, The Journal of Physical Chemistry C 124 (34) (2020) $18532-18543$.

[32] A. Phan, T.A. Ho, D.R. Cole, A. Striolo, Molecular Structure and Dynamics in Thin Water Films at Metal Oxide Surfaces: Magnesium, Aluminum, and Silicon Oxide Surfaces, The Journal of Physical Chemistry C 116 (30) (2012) $15962-$ 15973.

[33] A. Phan, D.R. Cole, A. Striolo, Liquid Ethanol Simulated on Crystalline Alpha Alumina, The Journal of Physical Chemistry B 117 (14) (2013) 3829-3840.

[34] S.B. Badmos, A. Striolo, D.R. Cole, Aqueous Hydrogen Sulfide in Slit-Shaped Silica Nanopores: Confinement Effects on Solubility, Structural, and Dynamical Properties, The Journal of Physical Chemistry C 122 (26) (2018) $14744-14755$.

[35] H. Fan, D.E. Resasco, A. Striolo, Amphiphilic Silica Nanoparticles at the Decane-Water Interface: Insights from Atomistic Simulations, Langmuir 27 (9) (2011) 5264-5274.

[36] A. Khedr, A. Striolo, DPD Parameters Estimation for Simultaneously Simulating Water-Oil Interfaces and Aqueous Nonionic Surfactants, J Chem Theory Comput 14 (12) (2018) 6460-6471.

[37] J.L.F. Abascal, E. Sanz, R.G. Fernandez, C. Vega, A potential model for the study of ices and amorphous water: TIP4P/Ice, J Chem Phys 122 (23) (2005) 234511.

[38] M.R. Walsh, C.A. Koh, E.D. Sloan, A.K. Sum, D.T. Wu, Microsecond Simulations of Spontaneous Methane Hydrate Nucleation and Growth, Science 326 (5956) (2009) 1095-1098.

[39] A. Phan, H. Schlösser, A. Striolo, Molecular mechanisms by which tetrahydrofuran affects $\mathrm{CO} 2$ hydrate Growth: Implications for carbon storage, Chem Eng J 418 (2021) 129423.

[40] M.G. Martin, J.I. Siepmann, Transferable potentials for phase equilibria. 1. United-atom description of n-alkanes, J Phys Chem B 102 (14) (1998) 25692577.

[41] J.M. Wang, R.M. Wolf, J.W. Caldwell, P.A. Kollman, D.A. Case, Development and testing of a general amber force field, J Comput Chem 25 (9) (2004) 11571174.

[42] D.A. Case, V. Babin, J.T. Berryman, R.M. Betz, Q. Cai, D.S. Cerutti, T.E.I. Cheatham, T.A. Darden, R.E. Duke, H. Gohlke, A.W. Goetzm, S. Gusarov, N. Homeyer, P. Janowski, J. Kaus, I. Kolossváry, A. Kovalenko, T.S. Lee, S. LeGrand, T.L. Luchko, R., B. Madej, K.M. Merz, F. Paesani, D.R. Roe, A. Roitberg, C. Sagui, R. Salomon-Ferrer, G. Seabra, C.L. Simmerling, W. Smith, J. Swails, R.C. Walker, J. Wang, R.M. Wolf, X. Wu, P.A. Kollman, AMBER 14, University of California, San Francisco, 2014

[43] J.W. Eastwood, R.W. Hockney, D.N. Lawrence, P3m3dp - the 3-Dimensional Periodic Particle-Particle-Particle-Mesh Program, Comput Phys Commun 19 (2) (1980) 215-261.

[44] M.P. Allen, D.J. Tildesley, Computer Simulation of Liquids, Oxford University Press, Oxford, UK, 2004.

[45] M.J. Abraham, T. Murtola, R. Schulz, S. Páll, J.C. Smith, B. Hess, E. Lindahl, GROMACS: High performance molecular simulations through multi-level parallelism from laptops to supercomputers, SoftwareX 1 (2015) 19-25.

[46] T.T.B. Le, C. Divine-Ayela, A. Striolo, D.R. Cole, Effects of surface contamination on the interfacial properties of $\mathrm{CO} /$ water/calcite systems, Phys Chem Chem Phys 23 (34) (2021) 18885-18892.

[47] J. Zhang, F. Leroy, F. Müller-Plathe, Influence of Contact-Line Curvature on the Evaporation of Nanodroplets from Solid Substrates, Phys Rev Lett 113 (4) (2014) 046101.

[48] G. Scocchi, D. Sergi, C. D’Angelo, A. Ortona, Wetting and contact-line effects for spherical and cylindrical droplets on graphene layers: A comparative molecular-dynamics investigation, Phys Rev E 84 (6) (2011) 061602. 
[49] T.A. Ho, Y. Wang, A. Ilgen, L.J. Criscenti, C.M. Tenney, Supercritical CO2-induced atomistic lubrication for water flow in a rough hydrophilic nanochannel, Nanoscale 10 (42) (2018) 19957-19963.

[50] H. Barrio-Zhang, É. Ruiz-Gutiérrez, S. Armstrong, G. McHale, G.G. Wells, R. Ledesma-Aguilar, Contact-Angle Hysteresis and Contact-Line Friction on Slippery Liquid-like Surfaces, Langmuir 36 (49) (2020) 15094-15101.

[51] M. Chiricotto, G. Giunta, H.A. Karimi-Varzaneh, P. Carbone, Calculation of the work of adhesion of polyisoprene on graphite by molecular dynamics simulations, Soft Materials 18 (2-3) (2020) 140-149.

[52] A.P. Sgouros, G.G. Vogiatzis, G. Kritikos, A. Boziki, A. Nikolakopoulou, D. Liveris, D.N. Theodorou, Molecular Simulations of Free and Graphite Capped Polyethylene Films: Estimation of the Interfacial Free Energies, Macromolecules 50 (21) (2017) 8827-8844.

[53] T. Dreher, C. Lemarchand, N. Pineau, E. Bourasseau, A. Ghoufi, P. Malfreyt, Calculation of the interfacial tension of the graphene-water interaction by molecular simulations, The Journal of Chemical Physics 150 (1) (2019) 014703.

[54] T. Dreher, C. Lemarchand, L. Soulard, E. Bourasseau, P. Malfreyt, N. Pineau, Calculation of a solid/liquid surface tension: A methodological study, The Journal of Chemical Physics 148 (3) (2018) 034702.

[55] J.G. Kirkwood, F.P. Buff, The Statistical Mechanical Theory of Surface Tension, The Journal of Chemical Physics 17 (3) (1949) 338-343.

[56] A. Ghoufi, P. Malfreyt, D.J. Tildesley, Computer modelling of the surface tension of the gas-liquid and liquid-liquid interface, Chem Soc Rev 45 (5) (2016) 1387-1409.

[57] D. Duque, L.F. Vega, Some issues on the calculation of interfacial properties by molecular simulation, The Journal of Chemical Physics 121 (17) (2004) 86118617.

[58] R. Shuttleworth, The Surface Tension of Solids, Proceedings of the Physical Society. Section A 63 (5) (1950) 444-457.

[59] J.H. Irving, J.G. Kirkwood, The Statistical Mechanical Theory of Transport Processes. IV. The Equations of Hydrodynamics, The Journal of Chemical Physics 18 (6) (1950) 817-829.

[60] Z.M. Aman, K. Olcott, K. Pfeiffer, E.D. Sloan, A.K. Sum, C.A. Koh, Surfactant Adsorption and Interfacial Tension Investigations on Cyclopentane Hydrate, Langmuir 29 (8) (2013) 2676-2682.

[61] J.M. Vanegas, A. Torres-Sánchez, M. Arroyo, Importance of Force Decomposition for Local Stress Calculations in Biomembrane Molecular Simulations, J Chem Theory Comput 10 (2) (2014) 691-702.

[62] A. Torres-Sánchez, J.M. Vanegas, M. Arroyo, Examining the Mechanical Equilibrium of Microscopic Stresses in Molecular Simulations, Phys Rev Lett 114 (25) (2015) 258102.

[63] A. Torres-Sánchez, J.M. Vanegas, M. Arroyo, Geometric derivation of the microscopic stress: A covariant central force decomposition, Journal of the Mechanics and Physics of Solids 93 (2016) 224-239.

[64] D.Y. Kwok, A.W. Neumann, Contact angle measurement and contact angle interpretation, Adv Colloid Interfac 81 (3) (1999) 167-249.

[65] D.Y. Kwok, A.W. Neumann, Contact angle interpretation in terms of solid surface tension, Colloids and Surfaces A: Physicochemical and Engineering Aspects 161 (1) (2000) 31-48.

[66] T. Uchida, T. Ebinuma, S. Takeya, J. Nagao, H. Narita, Effects of Pore Sizes on Dissociation Temperatures and Pressures of Methane, Carbon Dioxide, and Propane Hydrates in Porous Media, The Journal of Physical Chemistry B 106 (4) (2002) 820-826.

[67] R. Anderson, M. Llamedo, B. Tohidi, R.W. Burgass, Experimental Measurement of Methane and Carbon Dioxide Clathrate Hydrate Equilibria in Mesoporous Silica, The Journal of Physical Chemistry B 107 (15) (2003) 3507-3514.

[68] Y. Seo, S. Lee, I. Cha, J.D. Lee, H. Lee, Phase Equilibria and Thermodynamic Modeling of Ethane and Propane Hydrates in Porous Silica Gels, The Journal of Physical Chemistry B 113 (16) (2009) 5487-5492.

[69] Z.M. Aman, C.A. Koh, Interfacial phenomena in gas hydrate systems, Chem Soc Rev 45 (6) (2016) 1678-1690.

[70] S.J. Hu, C.A. Koh, Interfacial Properties and Mechanisms Dominating Gas Hydrate Cohesion and Adhesion in Liquid and Vapor Hydrocarbon Phases, Langmuir 33 (42) (2017) 11299-11309.

[71] P.M. Naullage, A.A. Bertolazzo, V. Molinero, How Do Surfactants Control the Agglomeration of Clathrate Hydrates?, Acs Central Sci 5 (3) (2019) 428-439

[72] C.A. Ward, J. Wu, Effect of Adsorption on the Surface Tensions of Solid-Fluid Interfaces, The Journal of Physical Chemistry B 111 (14) (2007) 3685-3694.
[73] A. Georgiadis, G. Maitland, J.P.M. Trusler, A. Bismarck, Interfacial Tension Measurements of the $(\mathrm{H} 2 \mathrm{O}+\mathrm{n}$-Decane $+\mathrm{CO} 2)$ Ternary System at Elevated Pressures and Temperatures, Journal of Chemical \& Engineering Data 56 (12) (2011) 4900-4908

[74] A.A. Bertolazzo, P.M. Naullage, B. Peters, V. Molinero, The Clathrate-Water Interface Is Oleophilic, The Journal of Physical Chemistry Letters 9 (12) (2018) 3224-3231.

[75] B.C. Knott, V. Molinero, M.F. Doherty, B. Peters, Homogeneous Nucleation of Methane Hydrates: Unrealistic under Realistic Conditions, J Am Chem Soc 134 (48) (2012) 19544-19547.

[76] S. Zeppieri, J. Rodríguez, A.L. López de Ramos, Interfacial Tension of Alkane + Water Systems, Journal of Chemical \& Engineering Data 46 (5) (2001) 1086 1088.

[77] D.M. Spori, T. Drobek, S. Zürcher, M. Ochsner, C. Sprecher, A. Mühlebach, N.D. Spencer, Beyond the Lotus Effect: Roughness Influences on Wetting over a Wide Surface-Energy Range, Langmuir 24 (10) (2008) 5411-5417.

[78] K.H. Chu, R. Xiao, E.N. Wang, Uni-directional liquid spreading on asymmetric nanostructured surfaces, Nat Mater 9(5) (2010) 413-7

[79] M. Liu, Y. Zheng, J. Zhai, L. Jiang, Bioinspired Super-antiwetting Interfaces with Special Liquid-Solid Adhesion, Accounts Chem Res 43 (3) (2010) 368-377.

[80] H.G. Ozcelik, E. Satiroglu, M. Barisik, Size dependent influence of contact line pinning on wetting of nano-textured/patterned silica surfaces, Nanoscale 12 (41) (2020) $21376-21391$.

[81] A. Calvimontes, A thermodynamic approach to predict apparent contact angles on microstructures using surface polygonal maps, Soft Matter 10 (41) (2014) 8308-8323.

[82] M. Suttipong, B.P. Grady, A. Striolo, Self-assembled surfactants on patterned surfaces: confinement and cooperative effects on aggregate morphology, Phys Chem Chem Phys 16 (31) (2014) 16388-16398.

[83] M. Suttipong, B.P. Grady, A. Striolo, Surfactant Aggregates Templated by Lateral Confinement, The Journal of Physical Chemistry B 119 (17) (2015) 5467-5474.

[84] M. Suttipong, B.P. Grady, A. Striolo, Surfactants adsorption on crossing stripes and steps, Soft Matter 13 (4) (2017) 862-874.

[85] E. Fadeeva, S. Schlie-Wolter, B.N. Chichkov, G. Paasche, T. Lenarz, 5 Structuring of biomaterial surfaces with ultrashort pulsed laser radiation, in: R. Vilar (Ed.), Laser Surface Modification of Biomaterials, Woodhead Publishing, 2016, pp. 145-172.

[86] D.I. Yu, H.J. Kwak, C. Park, C. Choi, N.P. Sapkal, J. Hong, M.H. Kim, Wetting Criteria of Intrinsic Contact Angle To Distinguish between Hydrophilic and Hydrophobic Micro-/Nanotextured Surfaces: Experimental and Theoretical Analysis with Synchrotron X-ray Imaging, Langmuir 35 (10) (2019) 36073614.

[87] R. Shuttleworth, G.L.J. Bailey, The spreading of a liquid over a rough solid, Discussions of the Faraday Society 3 (1948) 16-22.

[88] S. Hu, Interfacial Properties of CH4/C2H6 Gas Hydrate Particles with Chemical Additives, Colorado School of Mines, Ann Arbor, 2019, p. 156.

[89] F. Wang, H. Wu, Molecular origin of contact line stick-slip motion during droplet evaporation, Sci Rep-Uk 5 (1) (2015) 17521.

[90] W. Xu, C.-H. Choi, From Sticky to Slippery Droplets: Dynamics of Contact Line Depinning on Superhydrophobic Surfaces, Phys Rev Lett 109 (2) (2012) 024504.

[91] T.G.M. van de Ven, Capillary Forces in Wet Paper, Ind Eng Chem Res 47 (19) (2008) 7250-7256.

[92] A. Dupré, P. Dupré, Théorie mécanique de la chaleur, Gauthier-Villars1869.

[93] Z. Chen, B. Liu, R. Manica, Q. Liu, Z. Xu, Interaction Between the Cyclopentane Hydrate Particle and Water Droplet in Hydrocarbon Oil, Langmuir 36 (8) (2020) 2063-2070.

[94] C. Hu, N.C. Garcia, R. Xu, T. Cao, A. Yen, S.A. Garner, J.M. Macias, N. Joshi, R.L. Hartman, Interfacial Properties of Asphaltenes at the Heptol-Brine Interface, Energ Fuel 30 (1) (2016) 80-87.

[95] J.H. Song, A. Couzis, J.W. Lee, Direct Measurements of Contact Force between Clathrate Hydrates and Water, Langmuir 26 (12) (2010) 9187-9190.

[96] C. Liu, M. Li, G. Zhang, C.A. Koh, Direct measurements of the interactions between clathrate hydrate particles and water droplets, Phys Chem Chem Phys 17 (30) (2015) 20021-20029.

[97] W. Lee, S. Baek, J.-D. Kim, J.W. Lee, Effects of Salt on the Crystal Growth and Adhesion Force of Clathrate Hydrates, Energ Fuel 29 (7) (2015) 4245-4254. 